\title{
Insight into cross-talk between intra-amoebal pathogens
}

\author{
Gregory Gimenez ${ }^{1 \dagger}$, Claire Bertelli ${ }^{2 \dagger}$, Claire Moliner ${ }^{1}$, Catherine Robert ${ }^{1}$, Didier Raoult ${ }^{1}$, Pierre-Edouard Fournier ${ }^{1 *}$ \\ and Gilbert Greub ${ }^{2 *}$
}

\begin{abstract}
Background: Amoebae are phagocytic protists where genetic exchanges might take place between amoebaresistant bacteria. These amoebal pathogens are able to escape the phagocytic behaviour of their host. They belong to different bacterial phyla and often show a larger genome size than human-infecting pathogens. This characteristic is proposed to be the result of frequent gene exchanges with other bacteria that share a sympatric lifestyle and contrasts with the genome reduction observed among strict human pathogens.

Results: We sequenced the genome of a new amoebal pathogen, Legionella drancourtii, and compared its gene content to that of a Chlamydia-related bacterium, Parachlamydia acanthamoebae. Phylogenetic reconstructions identified seven potential horizontal gene transfers (HGTs) between the two amoeba-resistant bacteria, including a complete operon of four genes that encodes an ABC-type transporter. These comparisons pinpointed potential cases of gene exchange between P. acanthamoebae and Legionella pneumophila, as well as gene exchanges between other members of the Legionellales and Chlamydiales orders. Moreover, nine cases represent possible HGTs between representatives from the Legionellales or Chlamydiales and members of the Rickettsiales order.
\end{abstract}

Conclusions: This study identifies numerous gene exchanges between intracellular Legionellales and Chlamydiales bacteria, which could preferentially occur within common inclusions in their amoebal hosts. Therefore it contributes to improve our knowledge on the intra-amoebal gene properties associated to their specific lifestyle.

\section{Background}

Free-living amoebae are environmental protozoa, which are important predators that contribute to the control of microbial communities [1]. They feed by phagocytosing bacteria and other large, energy-rich particles that are present in their environment [2,3]. Several microorganisms have adapted to become resistant to amoebal phagocytosis and are able to escape the phagocytic pathway and multiply within phagocytic vacuoles [4]. Certain bacteria that live naturally in amoebae have been proposed to be amoebal pathogens $[5,6]$. These organisms

\footnotetext{
* Correspondence: Pierre-edouard.fournier@univmed.fr; gilbert.greub@chuv. ch

† Contributed equally

'Unité des rickettsies, Faculté de Médecine, Université de la Méditerranée, Marseille, France URMITE CNRS-IRD UMR 6236, Faculté de Médecine, 27 boulevard Jean Moulin, 13385 Marseille Cedex 05, France

${ }^{2}$ Center for Research on Intracellular Bacteria, Institute of Microbiology, University of Lausanne and University Hospital, Bugnon 48, 1011 Lausanne, Switzerland

Full list of author information is available at the end of the article
}

are phylogenetically diverse and belong to Chlamydiae; $\alpha, \beta$ and $\gamma$-proteobacteria; and Bacteroidetes $[7,8]$. The similarities in the strategies used by phylogenetically distantly related intracellular pathogens are most easily explained by convergent evolution [9]. Recently, Schmitz-Esser et al. [10] reported 59 functional domains that are significantly enriched in amoeba-associated bacteria; half of these domains are more frequently associated with eukaryotic proteins. However, the genomic characteristics associated with an intra-amoebal lifestyle remain largely unknown.

To date, few bacterial genomes from natural pathogens of amoebae have been fully sequenced, including those from (i) three strains of Chlamydia-related bacteria, i.e., "Candidatus Protochlamydia amoebophila" [11], Parachlamydia acanthamoebae [12] and Waddlia chondrophila [13]; (ii) five strains of Legionella pneumophila (Philadelphia, Lens, Paris, Corby, and Alcoy) [14-17] and two strains of L. longbeachae [18]; (iii)
C Biomed Central

() 2011 Gimenez et al; licensee BioMed Central Ltd. This is an Open Access article distributed under the terms of the Creative Commons Attribution License (http://creativecommons.org/licenses/by/2.0), which permits unrestricted use, distribution, and reproduction in any medium, provided the original work is properly cited. 
Rickettsia bellii [19];(iv) and a member of Bacteroidetes, "Candidatus Amoebophilus asiaticus" [10].

Recently, the new Legionella species L. drancourtii, initially named Legionella-like amoebal pathogen 12 (LLAP-12), was described within an Acanthamoeba sp. [20]. This bacterial species, known to be a strict intracellular pathogen of amoebae, exhibits a genome that is distinctly larger than its closest relatives, Francisella tularensis and Coxiella burnetii [7]. Despite its strict intracellular lifestyle, P. acanthamoebae exhibits a 3-fold larger genome than members of the Chlamydia genus [12] which includes human and animal pathogens that do not grow in amoebae [21]. These characteristics contrast with the observed genome-size reduction that is associated with the transition from a free-living to an intracellular lifestyle [22,23]. It has been proposed that the larger genome size might be linked to the sympatric lifestyle of intra-amoebal bacteria and to gene exchange among amoeba-resistant microorganisms inside the amoeba or between these microorganisms and the amoeba itself $[7,24]$.

A major mechanism involved in genetic exchanges between amoeba-resistant bacteria and their host is the type IV secretion system (T4SS) $[25,26]$. This widespread system can be divided into three major subtypes: those that mediate DNA transfer (F-like system), and those that translocate proteins and nucleoprotein complexes (P- and I- like systems) [27]. All of the studied genomes of intra-amoebal bacteria, including Legionellae, R. bellii and Pr. amoebophila, exhibit a T4SS of differing subtypes $[14,15,17,19,28,29]$, except for $W$. chondrophila, in which no such system has been identified [13]. Interestingly, a partial F-like conjugative system has been identified in the draft genome of $P$. acanthamoebae [12].

In this study, we sequence the genome of the L. drancourtii strain LLAP-12 and compare it to the genome of the $P$. acanthamoebae strain Hall's coccus in order to identify genes that could have been exchanged between these two amoebal pathogens and, more widely, between Chlamydiae and Legionellae.

\section{Results}

Dirty genomes of $L$. drancourtii and $P$. acanthamoebae Sequencing reads from $L$. drancourtii strain LLAP-12 obtained from 454 pyrosequencing were assembled into 213 contigs that were further organized into 58 scaffolds (see Additional file 1, Materials). The genome had a total size of 4,062,386 bp and a G+C content of $39 \%$. A total of 3,965 protein-coding ORFs with an average size of 869 bp were identified and cover $84.7 \%$ of the genome. The published genome of the $P$. acanthamoebae strain Hall's coccus [12] comprises 93 contigs and has a total of $2,971,261$ bp with a $G+C$ content of $38 \%$. The
2,809 predicted genes have an average size of $943 \mathrm{bp}$ and encompass $89 \%$ of the genome. The genome of Legionella drancourtii and Parachlamydia acanthamoebae have been deposited in GenBank under accession number ACUL02000000 and NZ_ACZE00000000, respectively.

\section{Orthologues identification}

To identify orthologous genes that might have been acquired by lateral gene transfer, proteins of $P$. acanthamoebae and $L$. drancourtii were subjected to reciprocal BLASTP searches. A total of 508 orthologous proteins were identified as exhibiting a reciprocal similarity percentage $>30 \%$ and an alignment length coverage $>60 \%$ (Figure 1). Each orthologue was subjected to BLASTP homology searches against one amoebal and six bacterial genomes and was considered present if it fulfilled identical cutoffs for similarity and coverage. A clustering analysis according to the presence or absence of the orthologues in the different organisms was used to categorize the genes into eight different categories, as illustrated in the heatmap in Figure 2. The clustering of bacteria in the horizontal top tree indicates that

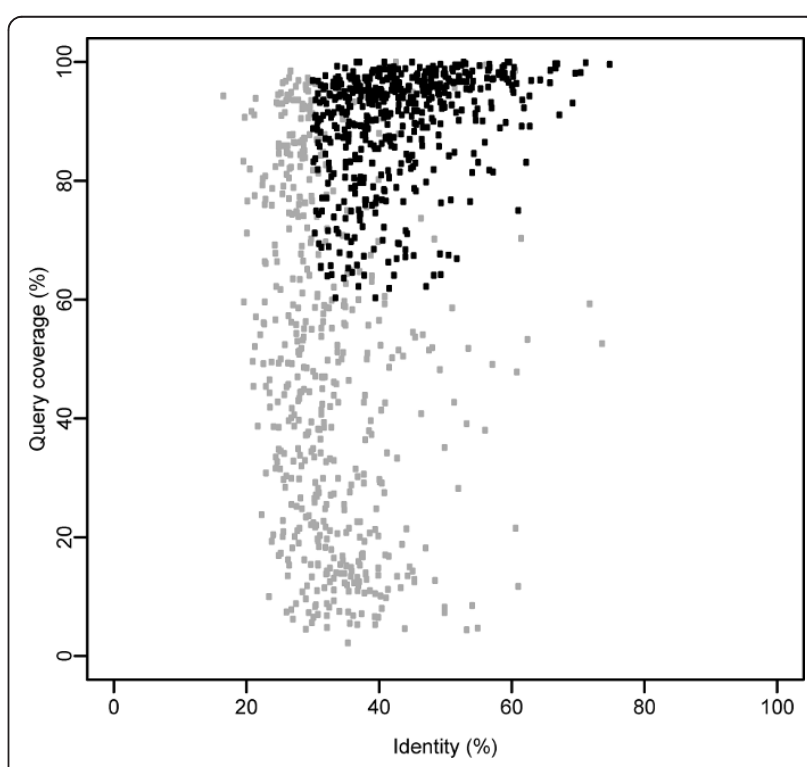

Figure 1 Definition of orthologous proteins. Representation of the identity (\%) and coverage (\%) of the query alignment that results from a BLASTP search of $P$. acanthamoebae versus $L$. drancourtii. Of the 1023 proteins showing reciprocal best blast hits, 508 exhibited a similarity greater than $30 \%$ and an alignment coverage longer than $60 \%$ of the query (black) and were defined as orthologues. Of the remaining 515 proteins (gray), 53 did not fulfill the same criteria in the reciprocal analysis and, therefore were discarded, although they appear on this figure as passing the cutoffs. The correlation coefficient between both of the reciprocal BLAST analyses was 0.97 and 0.80 for the percentage identity and the percentage coverage, respectively. 


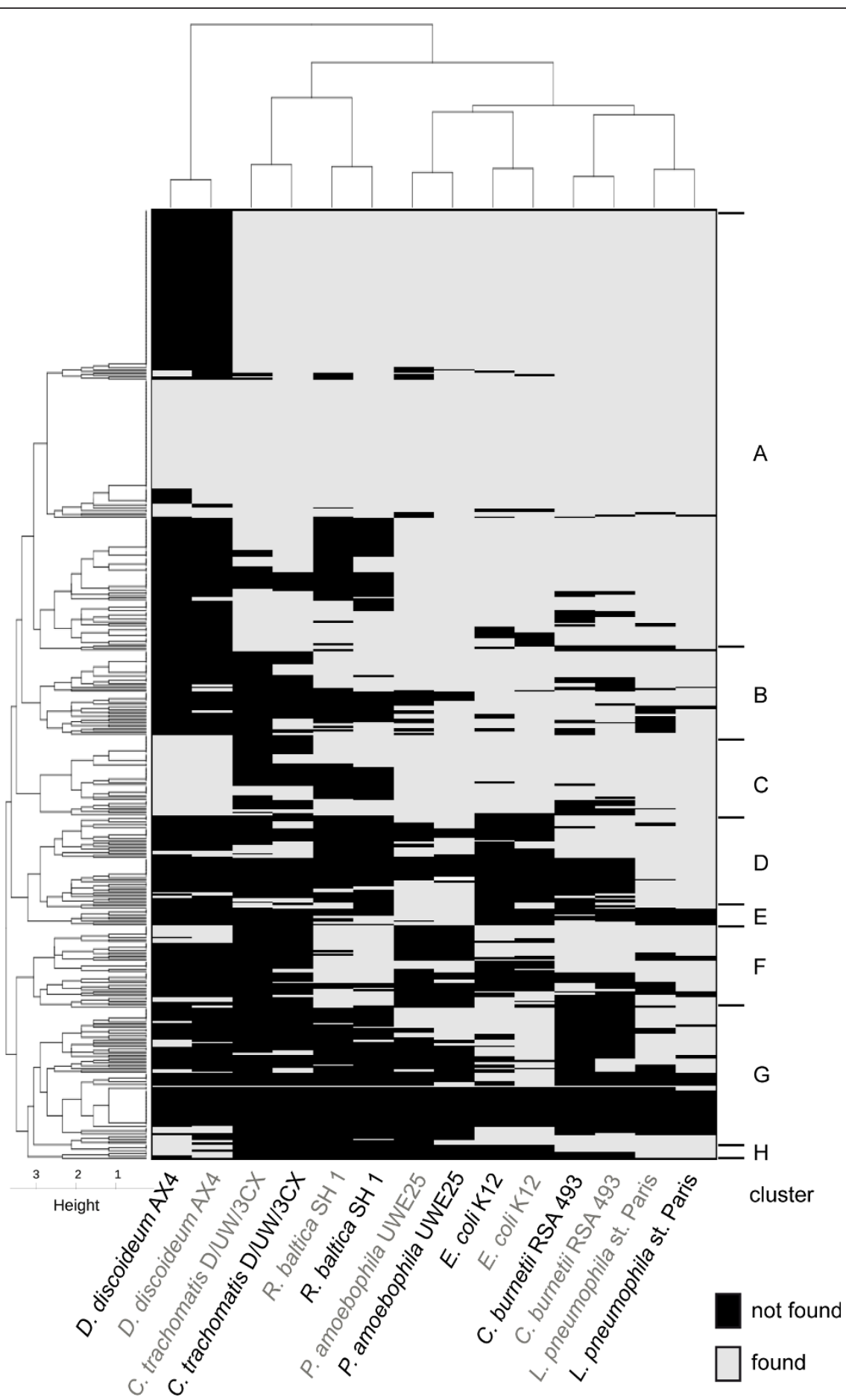

Figure 2 Gene clusters. Orthologous genes were subjected to BLASTP against seven other organisms, colored in gray and black when using Parachlamydia and Legionella proteins as queries, respectively. Genes were clustered according to their presence (gray) or absence (black) in the six related bacteria and the amoebal species, D. discoideum. The genes form 8 major groups at a height of $3(\mathrm{~A}$ to $\mathrm{H})$.

consistent results were obtained when using $P$. acanthamoebae or L. drancourtii orthologues as queries against the other genomes. Cluster A consists of core genes that are present in nearly all bacteria. Clusters B and C represent core genes generally absent in C. trachomatis and/or R. baltica but respectively absent and present in the amoeba Dictyostelium. The absence of an ortholog in C. trachomatis is likely due to the genome reduction 
that arose in the Chlamydia genus, whereas the absence of an ortholog in $R$. baltica might reflect the divergence of the species. The remaining clusters are made of differently conserved genes. Genes in cluster D are mainly present in Pr. amoebophila, L. pneumophila, and in C. burnetii. The small cluster E contains genes mainly present in Pr. amoebophila. Genes in cluster F are mostly present in L. pneumophila, C. burnetii and R. baltica and to a lesser extend in E. coli. Cluster G is formed of two subsets: one subset of genes absent from all compared genomes, and another subset containing genes present in Pr. amoebophila and E. coli. Cluster H mainly represents genes encoded by the gamma-proteobacteria and the amoeba Dictyostelium but absent from Chlamydiales and Planctomycetales. The genes were classified according to the number of hits obtained against each of the six bacterial genomes used (Table 1).

\section{Conservation of orthologous genes}

Core gene identification: Of the 508 orthologues, 208 were found in the genomes of Pr. amoebophila, C. trachomatis, R. baltica, L. pneumophila, C. burnetii, and E. coli (score 6; super-cluster A in Figure 2 and Additional file 2, Table S1). Of these orthologues, 92 coding sequences $(44 \%)$ were considered essential genes [30] and, notably, encode for 32 ribosomal proteins and 11 tRNA synthetases. In addition, 80 genes were present in five of the six screened genomes (score 5; Additional file 3, Table S2), of which 13 (16\%) belonged to the minimal gene set. These sequences included 9 ribosomal proteins and 3 tRNA synthetases due to their borderline values for identity and alignment coverage. Of these 80 coding sequences, 26 (33\%) were absent from the C. trachomatis genome, reflecting the important genome reduction that has occurred in this bacterial clade, especially among genes encoding for metabolic functions (12 genes belong to COGs C, E, F, G or H). In addition, 38 genes $(48 \%)$ were absent from the $R$. baltica genome, probably as a result of its greater phylogenetic distances to Parachlamydia and Legionella than the other bacteria screened. Only 9, 6, 1 and 0 genes were missing in the genomes of C. burnetii, E. coli, Pr. amoebophila and $L$. pneumophila, respectively. Finally, 77 genes were present in four of the six studied genomes (score 4; Additional file 4, Table S3) and 4 of them (5\%) belonged to the minimal gene set [30]. All of the coding sequences with a score of 4 or above were considered to be common genes found in most bacteria and were therefore not further investigated for potential HGT.

Accessory orthologues: Of the 143 remaining genes, 59 were identified in three screened bacterial species (score 3; Additional file 5, Table S4). There was no obvious logical pattern of distribution among the bacteria because no genes were common to the three amoebal pathogens included in the analysis (L. pneumophila, $C$. burnetii, and Pr. amoebophila), and only ten coding sequences were common to $\gamma$-proteobacteria (L. pneumophila, C. burnetii, and E. coli). Moreover, bacteria belonging to the Chlamydiae-Planctomycetes superphylum (Pr. amoebophila, C. trachomatis, and R. baltica) encoded for no common gene in this category. Thirtyseven orthologues exhibited homologues in two species (score 2; Additional file 6, Table S5). Unsurprisingly, $L$. pneumophila contained $76 \%$ of these genes indicating its close phylogenetic distance to L. drancourtii. In contrast, none of these genes were found only in bacteria belonging to the Chlamydiales order (Pr. amoebophila and $C$. trachomatis), while 12 genes were only found in bacteria belonging to the Legionellales order (L. pneumophila and C. burnetii).

In addition, 27 genes harbored similarities to a single species (score 1; Additional file 7, Table S6): L. pneumophila (15), E. coli (8) or Pr. amoebophila (4). Finally, as visualized in Figure 2 (cluster G), 20 genes were only present in $P$. acanthamoebae and $L$. drancourtii (score 0; Additional file 8, Table S7). Only one of these was

Table 1 Orthologous gene classification

\begin{tabular}{|c|c|c|c|c|c|c|c|}
\hline \multirow[b]{2}{*}{ Score } & \multirow[b]{2}{*}{$\begin{array}{l}\text { No. of } \\
\text { genes }\end{array}$} & \multicolumn{3}{|c|}{ Related to $L$. drancourtii } & \multicolumn{3}{|c|}{ Related to $P$. acanthamoebae } \\
\hline & & $\begin{array}{c}\text { Legionella } \\
\text { pneumophila }\end{array}$ & $\begin{array}{l}\text { Coxiella } \\
\text { burnetii }\end{array}$ & $\begin{array}{c}\text { Escherichia } \\
\text { coli }\end{array}$ & $\begin{array}{l}\text { Protochlamydia } \\
\text { amoebophila }\end{array}$ & $\begin{array}{l}\text { Chlamydia } \\
\text { trachomatis }\end{array}$ & $\begin{array}{c}\text { Rhodopirellula } \\
\text { baltica }\end{array}$ \\
\hline 0 & 20 & 0 & 0 & 0 & 0 & 0 & 0 \\
\hline 1 & 27 & 15 & 0 & 8 & 4 & 0 & 0 \\
\hline 2 & 37 & 28 & 16 & 10 & 12 & 0 & 8 \\
\hline 3 & 59 & 52 & 27 & 41 & 32 & 6 & 19 \\
\hline 4 & 77 & 76 & 60 & 62 & 58 & 16 & 36 \\
\hline 5 & 80 & 80 & 71 & 74 & 79 & 54 & 42 \\
\hline 6 & 208 & 208 & 208 & 208 & 208 & 208 & 208 \\
\hline
\end{tabular}

This table indicates the total number of proteins with a score of 0 to 6 based on their presence in the six bacteria when using $P$. acanthamoebae or $L$. drancourti orthologous proteins as queries for the BLASTP searches. The number of corresponding genes present in each bacterium is detailed in the following columns.

The bacteria in the first three columns are decreasingly related to $L$. drancourtii, andthose indicated in the last three columns exhibit diminishing relatedness to P. acanthamoebae. 
also present in $D$. discoideum: a conserved protein of unknown function that was also encoded only in the Corby strain of L. pneumophila and not in the Paris strain of L. pneumophila that was used in BLAST analyses. Interestingly, this category included an operon for histidine biosynthesis, which was composed of four genes $(378,379,442$, and 443$)$, that was only found in $P$. acanthamoebae and the recently sequenced W. chondrophila [13] but not in the more closely-related $P r$. amoebophila. The corresponding genes in $L$. drancourtii were encoded at two different genomic regions and the four genes exhibited divergent phylogenies that render an acquisition of the entire operon from Legionella implausible.

\section{Horizontal gene transfers}

To identify potential gene transfers, the 143 genes with scores of 0 to 3 were subjected to phylogenetic reconstruction using the 20 best BLAST hits from each $P$. acanthamoebae and $L$. drancourtii orthologues. The topology of the trees deviated from the expected taxonomic distribution of representatives from the Chlamydiales and Legionellales orders in 37 cases that are detailed in Table 2. Overall, both $P$. acanthamoebae and L. drancourtii genes clustered together in 7 phylogenies (Additional file 9, Figure S1). Interestingly, four of these genes (ID 127, 128, 129, and 130) were organized in an operon that encodes for a permease and a membrane anchored ATP-binding protein of an ABC-2 type transporter, an HlyD family secretion protein and an outer membrane efflux protein (Figure 3). Similar systems that are widespread among gram-negative bacteria such as $E$. coli, Serratia marcescens and Pseudomonas aeruginosa form a specialized secretory system for proteins, such as pore-forming toxins, proteases, lipases and S-layer proteins [31]. Among the six bacteria screened, a partial operon was found only in E. coli, where the first three coding sequences had strong homologues, but the fourth gene encoding for the outer membrane efflux protein exhibited a homologue at a more distant locus. Notably, the tree topology was similar for all of these proteins (Figure 3) suggesting that the entire operon was exchanged between $L$. drancourtii and $P$. acanthamoebae in a single event.

L. drancourtii never clustered with several members of the Chlamydiales order. However, P. acanthamoebae branched with the Legionellales clade in $10 \%$ of the trees (15/143) (Additional file 10, Figure S2 A-O). A good example of this clustering is a hypothetical protein (ID 247), where P. acanthamoebae clustered with a branch containing Francisella and two Legionella species, suggesting that a transfer occurred before the divergence of the Legionella and Francisella genera. In three additional instances (ID 400, 422, and 466), $P$. acanthamoebae clustered specifically with L. pneumophila and far from other Legionellales (Additional file 10, Figure S2 P-R) suggesting a transfer between these two species. These genes were located on different contigs and encoded a permease, an HlyD family protein belonging to an $\mathrm{ABC}$-transporter, and a putative transcriptional regulator. An example of a protein wrongly considered only to be present in $L$. drancourtii and $P$. acanthamoebae is an acetyltransferase (ID 191) that has a borderline similarity value of $29.4 \%$ with $L$. pneumophila. In fact, the corresponding gene was found in several members of the Rickettsiales order (Rickettsia, Wolbachia, and Orientia), in Legionella species and in P. acanthamoebae. We hypothesize that this protein's presence in Parachlamydia results from a HGT with an ancestor of the Legionellales order.

Furthermore, the Legionellales and Chlamydiales orders were directly related in the tree that was inferred from four genes (ID 90, 207, 263, 282) that encode for a multidrug resistance protein, a 7-dehyrocholesterol reductase, a protein that protects DNA during starvation and a conserved hypothetical protein, respectively (Additional file 11, Figure S3). The gene involved in the protection against oxidative stress (dpsA, ID 263) presented a particular topology. A phylogenetic tree inferred from an analysis with the 20 best BLAST hits indicated that this gene was recently exchanged between Rickettsiales and Legionellales and that an exchange between Legionellae and Chlamydiae had occurred earlier (Figure 4). Notably, two different copies of this gene were also present in the cyanobacterium Synechococcus, one of which branched closely to Chlamydiales, suggesting an exchange with an ancestral Chlamydiae, and another copy clustered with other cyanobacterial genomes and $\beta$-proteobacteria.

The phylogenetic analysis identified 9 cases of potential gene transfer between $P$. acanthamoebae or L. drancourtii and Rickettsia, Orientia, Wolbachia and Ehrlichia (Additional file 12, Figure S4). Of these cases, five genes (ID $243,263,387,388$, and 390) may have been horizontally transferred between L. drancourtii and Rickettsia, including a beta-lactamase (ID 243). The analysis of the ID 387 encoded hypothetical protein clustered $P$. acanthamoebae, Legionellales and Orientia together. The close relationship between $L$. drancourtii and Orientia suggests a transfer to the latter species (Figure 5). The tree of the aminoglycoside transferase (ID 388) illustrates a complex situation where all of the intracellular bacteria were grouped in a single cluster, suggesting a common ancestral source for this gene. The ATPase and permease subunits of an ABCtype transporter (ID 390) exhibited the remarkable phylogenetic pattern of being nearly exclusive to intracellular species. However, this tree exhibited an atypical configuration that showed no monophyly among Legionella and 
Table 2 Potential horizontal gene transfers identified

\begin{tabular}{|c|c|c|c|c|c|c|c|c|}
\hline Score & $\begin{array}{l}\text { PAH- } \\
\text { LLAP }\end{array}$ & $\begin{array}{c}\text { PAH- } \\
\text { Legionellales } \\
\end{array}$ & $\begin{array}{l}\text { Legionellales- } \\
\text { Chlamydiales }\end{array}$ & $\begin{array}{l}\text { PAH/LLAP- } \\
\text { Rickettsiales }\end{array}$ & $\begin{array}{l}\text { PAH/LLAP- } \\
\text { Others }\end{array}$ & Gene product & $\begin{array}{c}\text { COG } \\
\text { accession }\end{array}$ & $\begin{array}{l}\text { COG } \\
\text { group }\end{array}$ \\
\hline \multirow[t]{7}{*}{0} & 101 & & & & & Hypothetical protein & & \\
\hline & 158 & & & & & Choloylglycine hydrolase & COG3049 & M \\
\hline & & 191 & & 191 PAH-Rickettsiales & & Acetyltransferase & COG0454 & KR \\
\hline & & 192 & & 192 PAH-Wolbachia & & $\begin{array}{l}\text { Aminoglycoside phosphotransferase } \\
\text { family protein }\end{array}$ & COG3173 & $\mathrm{R}$ \\
\hline & 473 & & & & & putative phosphotransferase & $\operatorname{cog} 2334$ & R \\
\hline & & 475 & & & & Hypothetical protein & COG0500 & QR \\
\hline & & 492 & & & & $\begin{array}{l}\text { Toluene efflux pump outer membrane } \\
\text { protein }\end{array}$ & COG1538 & MU \\
\hline \multirow[t]{9}{*}{1} & & 96 & & & & Conserved hypothetical protein & COG0599 & S \\
\hline & 127 & & & & & $\begin{array}{c}\text { ABC-type multidrug transporter, } \\
\text { permease }\end{array}$ & COG0842 & V \\
\hline & 128 & & & & & $\begin{array}{l}\text { ABC-type multidrug transporter, ATP- } \\
\text { binding protein }\end{array}$ & COG1131 & V \\
\hline & 129 & & & & & HlyD family secretion protein & COG0845 & M \\
\hline & & & & 243 LLAP-Rickettsia & & Beta-lactamase & $\operatorname{COG} 2602$ & V \\
\hline & & 246 & & & & Acetyltransferase, GNAT family & COG0454 & KR \\
\hline & & 247 & & & & Hypothetical protein & COG2132 & Q \\
\hline & & & 282 & & & Conserved hypothetical protein & COG4804 & S \\
\hline & & 387 & & $\begin{array}{c}387 \text { PAH- } \\
\text { Legionellales-Orientia }\end{array}$ & & Hypothetical protein & COG0500 & $Q R$ \\
\hline \multirow[t]{10}{*}{2} & & 11 & & & & Succinylarginine dihydrolase & COG3724 & $E$ \\
\hline & & 86 & & & & Metallo-beta-lactamase family protein & COG1234 & $\mathrm{R}$ \\
\hline & & 166 & & & & Multidrug resistance protein & COG0477 & GEPR \\
\hline & & & 207 & & $\begin{array}{l}207 \text { LLAP- } \\
\text { Naegleria }\end{array}$ & 7-dehydrocholesterol reductase & $\operatorname{COG} 2020$ & O \\
\hline & & 301 & & & & Acyl-CoA dehydrogenase & COG1960 & । \\
\hline & & & & 388 LLAP-Rickettsia & & Aminoglycoside $N\left(6^{\prime}\right)$-acetyltransferase & COG1670 & J \\
\hline & & $400^{*}$ & & & & Putative transcriptional regulator & COG0583 & K \\
\hline & & 421 & & & & Putative outer membrane efflux protein & COG1538 & $M U$ \\
\hline & & & & & $\begin{array}{c}458 \\
\text { Chlamydiales- } \\
\text { Eukaryotes }\end{array}$ & $\begin{array}{l}\text { Alpha keto acid dehydrogenase } \\
\text { complex E1 component alpha subunit }\end{array}$ & COG1071 & C \\
\hline & & 474 & & & & $\begin{array}{c}\text { DNA protection during starvation } \\
\text { protein }\end{array}$ & COG0783 & P \\
\hline \multirow[t]{11}{*}{3} & & & 90 & & & Multidrug resistance protein B homolog & COG0477 & GEPR \\
\hline & 130 & & & & & $\begin{array}{c}\text { Solvent efflux pump outer membrane } \\
\text { protein }\end{array}$ & COG1538 & $M U$ \\
\hline & & & & $\begin{array}{l}134 \text { Chlamydiales- } \\
\text { Ehrlichia/Wolbachia }\end{array}$ & & $\begin{array}{c}\text { Proton/sodium-glutamate symport } \\
\text { protein }\end{array}$ & COG1301 & C \\
\hline & & & & & $\begin{array}{l}208 \text { LLAP-A. } \\
\text { asiaticus }\end{array}$ & PEBP family protein & COG1881 & R \\
\hline & & & & 211 PAH-Rickettsia & & $\begin{array}{c}\text { Putative 6-pyruvoyl tetrahydrobiopterin } \\
\text { synthase }\end{array}$ & COG0720 & $\mathrm{H}$ \\
\hline & & & & & $\begin{array}{l}240 \text { LLAP- } \\
\text { Eukaryotes }\end{array}$ & Hypothetical protein & COG1092 & R \\
\hline & & & 263 & 263 LLAP-Rickettsia & & $\begin{array}{l}\text { DNA protection during starvation } \\
\text { protein } 2\end{array}$ & COG0783 & P \\
\hline & & & & $\begin{array}{l}390 \text { Legionellales- } \\
\text { Rickettsiales }\end{array}$ & & $\begin{array}{c}\text { ABC-type transporter, permease and } \\
\text { ATPase subunit }\end{array}$ & COG1132 & V \\
\hline & & 413 & & & & Cation-transporting ATPase & $\operatorname{COG} 2217$ & $P$ \\
\hline & & $422^{*}$ & & & & $\begin{array}{l}\text { Inner membrane transport permease } \\
\text { YbhR }\end{array}$ & COG0842 & V \\
\hline & & $466^{*}$ & & & & Methylisocitrate lyase & $\operatorname{COG} 2513$ & G \\
\hline
\end{tabular}

* clustering with Legionella pneumophila only, PAH = Parachlamydia acanthamoebae, LLAP = Legionella drancourtii

This table presents the classification by score and ID numbers of the $L$. drancourtii and $P$. acanthamoebae orthologues that exhibit a phylogenetic reconstruction suggesting horizontal gene transfer events. The gene product annotation, COG accession number and COG group are indicated for each gene. 


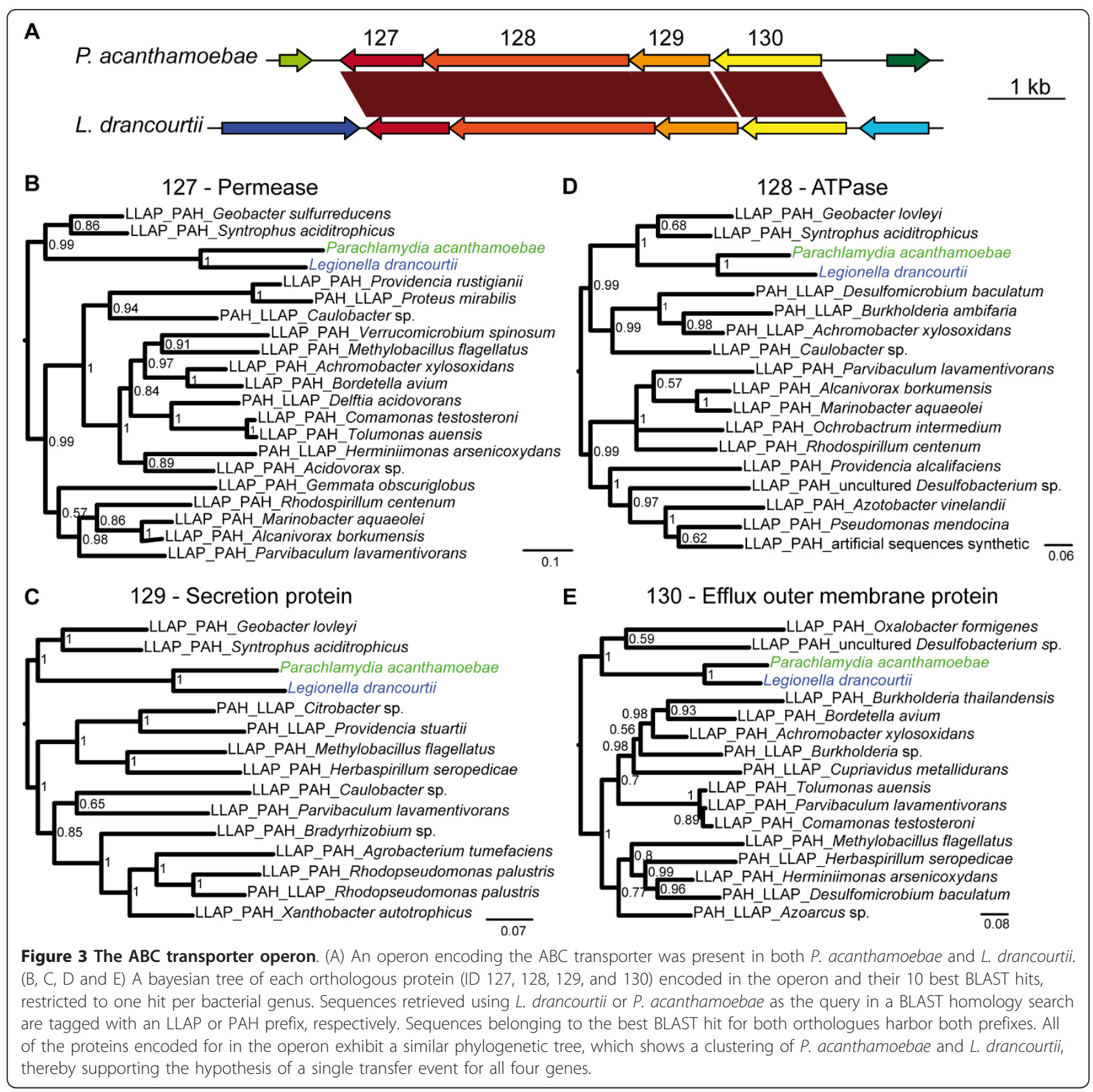

Parachlamydiaceae. Indeed, L. drancourtii clustered with L. pneumophila str. Paris and Parachlamydia, whereas another gene from L. pneumophila clustered with Protochlamydia. This topology suggests the presence of multiple paralogues within the L. pneumophila genome. We hypothesize that duplication events first occurred in $L$. pneumophila and that one paralogue was subsequently horizontally transferred to Protochlamydia and another paralogue to Parachlamydia. Finally, the tree for a proton/ sodium-glutamate symport protein (ID 134) suggests an exchange between Chlamydiales and an ancestral Rickettsiales (Figure 5).
In three cases (ID 207, 240 and 458), similarity searches retrieved eukaryotic proteins matching $L$. drancourtii and $P$. acanthamoebae proteins, including a 7-dehydrocholesterol reductase and a conserved hypothetical protein (ID 207 and 240, respectively) from L. drancourtii that seem closer to eukaryotes than to bacteria with the exception of L. longbeachae and a pyruvate dehydrogenase E1 alpha subunit (ID 458) (Additional file 13, Figure S5). Interestingly, BLAST searches with the 7-dehydrocholesterol reductase retrieved a protein from the amoeba Naegleria gruberi that clusters with L. drancourtii in the phylogenetic tree. In a recent study, this gene 


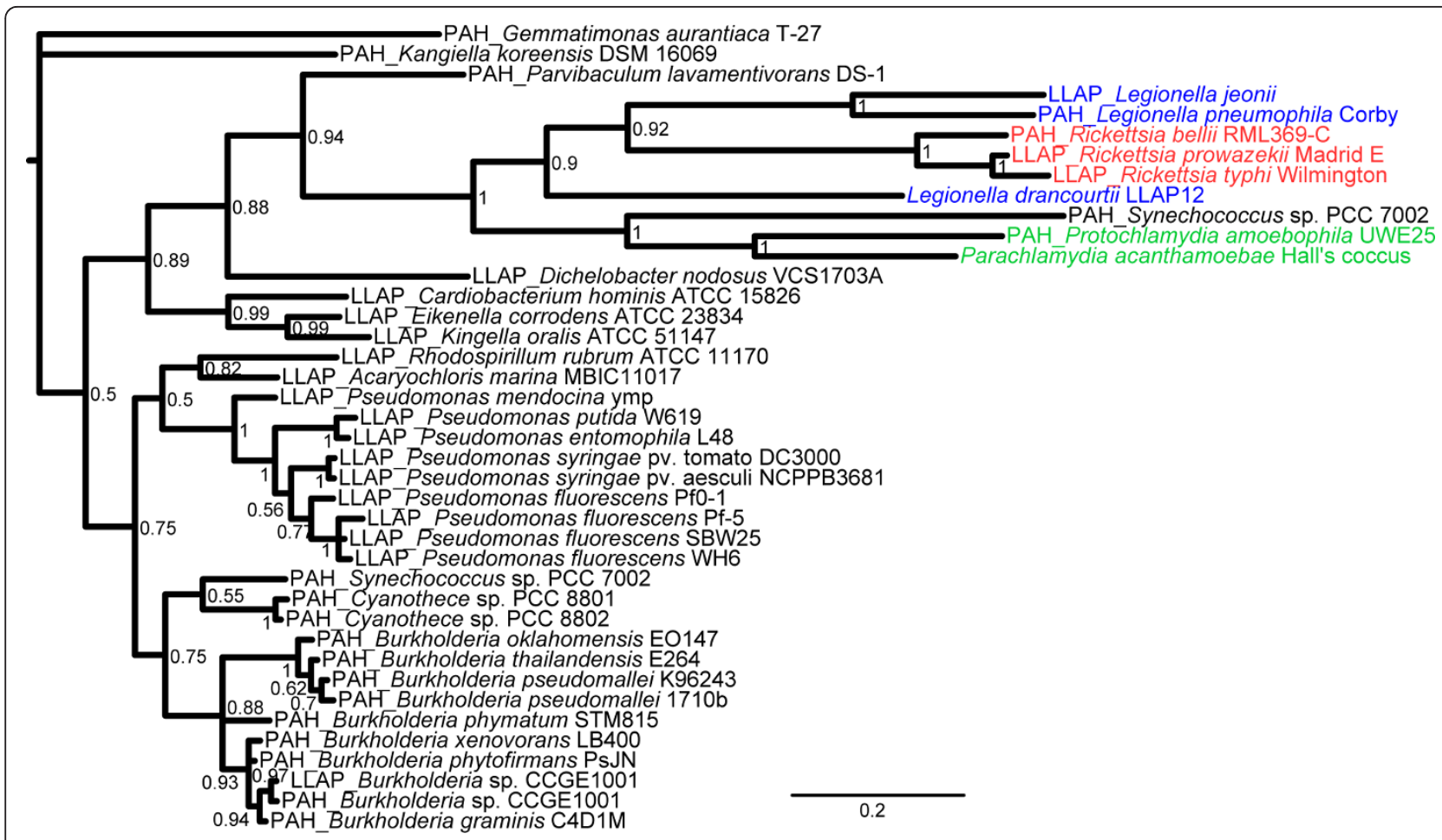

Figure 4 dpsA phylogeny. The bayesian tree for dpsA (ID 263), a protein involved in protection against oxidative stress, and the 20 best BLAST hits shows the clustering of proteins from Rickettsia with Legionella species and, to a lesser extent, with Parachlamydiaceae and Synechococcus, a cyanobacterium. Sequences retrieved using $L$. drancourtii or $P$. acanthamoebae as the query in a BLAST homology search are tagged with an LLAP or PAH prefix, respectively. Sequences belonging to the best BLAST hits for both orthologues harbor both prefixes.

was proposed to have been acquired from viridiplantae [32], but this earlier phylogenetic reconstruction did not include Naegleria. Finally, two widely conserved enzymes (ID 288 and 289, respectively) that encode for a malate synthase and an isocitrate lyase are syntenic in both genomes. A phylogenetic reconstruction with their twenty best BLAST hits and amoebae showed that the malate synthase of $L$. drancourtii clustered with Dictyostelium discoideum, suggesting a gene exchange from a Legionella ancestor to amoebae, as previously reported [33].

The genomes of $L$. drancourtii and $P$. acanthamoebae harbor respectively 45 and 8 insertion sequences (ISs), a number probably underestimated due to the likely presence of such elements in gaps of the genomic sequence. One IS of type ISNpu13 is located $2000 \mathrm{bp}$ upstream the gene LGD_5795 (ID 246) that encodes an acetyltransferase. There were no ISs close to other genes identified as potentially acquired by HGT (Table 2).

The genomic nucleotide composition, notably the GC content and codon usage, varies widely among the organisms. As a consequence, regions that were exchanged recently present an imprinting with the donor's characteristics. However, mutational pressures tend to adapt the compositional features of foreign
DNA to that of its host over time, thus making the transferred region indistinguishable [34]. In our analysis, the GC content of the 37 genes suspected to have been acquired by HGT based on phylogeny did not show a marked difference in the GC content compared to the rest of the Parachlamydia or Legionella genes (Additional file 14, Figure S6). The mean GC content for Parachlamydia and Legionella is respectively $38.5 \%$ and $39 \%$ for all genes and, more precisely, $33.7 \%$ and $32.6 \%$ at the third codon position, which is less constrained for the conservation of amino acid properties. An interesting case was the operon that encodes an $A B C$ transporter that showed the highest GC content, exhibiting values between $41 \%$ and $45 \%$ in $P$. acanthamoebae. In contrast, the GC content of this operon in $L$. drancourtii was less distinguishable from the mean gene $\mathrm{GC}$ values. This finding suggests that $P$. acanthamoebae acquired this operon by HGT with a partner that has a higher GC content than $L$. drancourtii.

Most exchanged genes encode poorly characterized functions represented by COG category R. However, several genes that belong to the overrepresented categories $\mathrm{R}, \mathrm{V}, \mathrm{M}$ and $\mathrm{P}$ encode putative functions linked to the transport of various molecules and resistance to toxic compounds. 


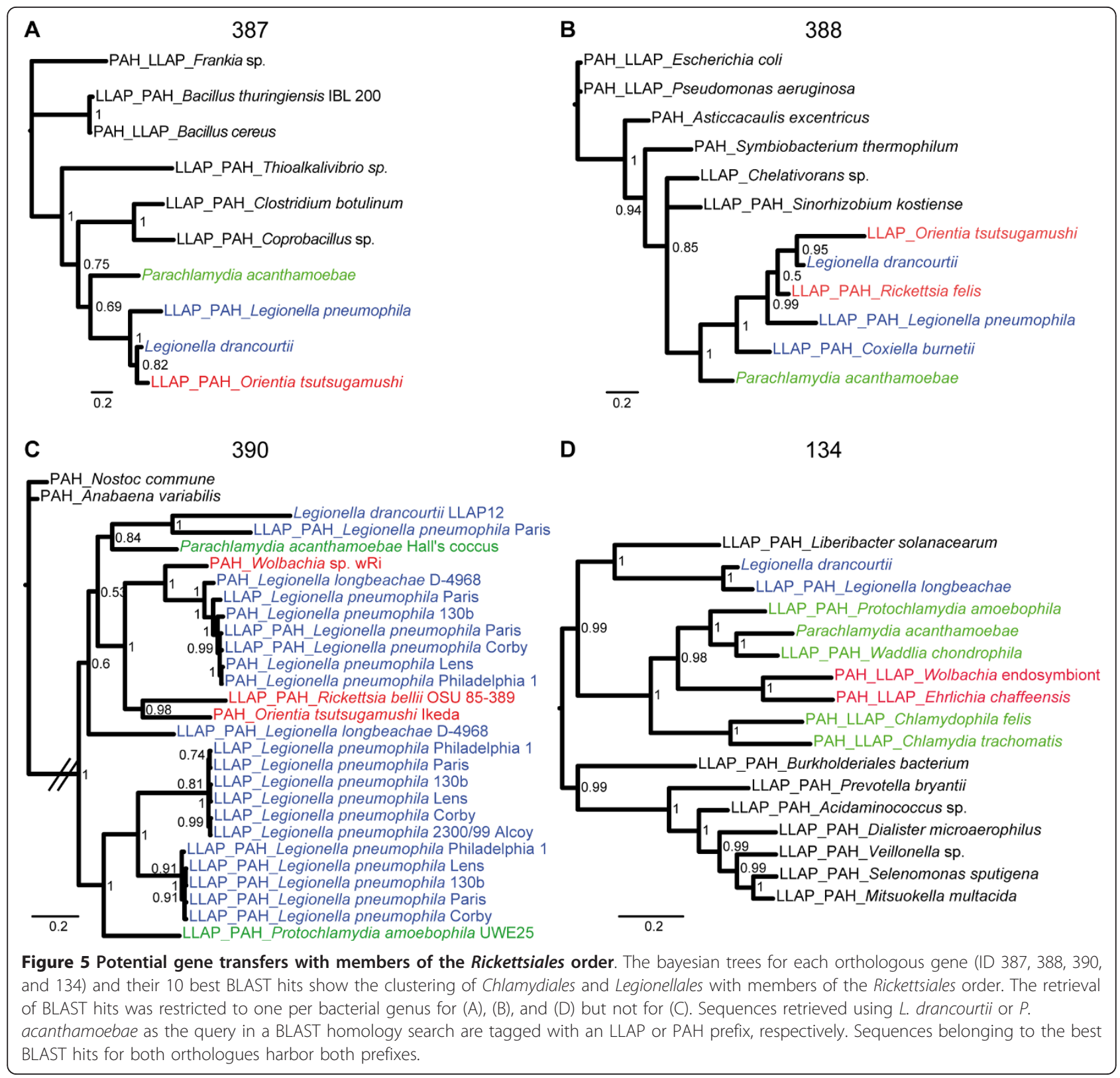

\section{Discussion}

In this study, the genes in the draft genomes of $P$. acanthamoebae and L. drancourtii were compared; we identified 508 orthologues, which were subsequently searched for in increasingly distantly related organisms. Although limited by the small number of related organisms that we used to define conserved core genes and less-conserved genes, our approach allowed us efficiently to discard $72 \%$ of the orthologues that are widespread within bacteria. The remaining $28 \%$ of the genes were submitted to phylogenetic reconstructions, which enabled us to detect 7 putative HGTs between $P$. acanthamoebae and L. drancourtii. In addition, 18 genes were potentially transferred from Legionellales to $P$. acanthamoebae, whereas no genes were transferred from Chlamydiales to L. drancourtii. As expected, half of these potential HGTs encode for transporters and resistance mechanisms that could be of particular importance for the bacteria to resist to the microbicidal defences of its amoebal host and to survive with high concentrations of toxic compounds. The remaining HGTs encode for metabolic functions or members of poorly characterized protein families. Their importance and their role regarding the amoebal pathogenicity of $L$. drancourtii and $P$. acanthamoebae remain to be determined. 
We acknowledge the fact that the stringent reciprocal identity and coverage cut-offs constitute one major limitation of this method, as they may falsely classify some genes as absent from one of the selected related species when they are, in fact, present. Genes that were probably acquired by HGT showed a large distribution in their percentage of identity, ranging from $30 \%$ to $71 \%$, but they all exhibited an alignment coverage greater than $80 \%$ indicating that a more stringent criterion could have been used (Additional file 15, Figure S7). The phylogenetic reconstructions enabled us to pinpoint such cases because closely related sequences were retrieved by BLAST searches. One major cause of these false negatives are borderline values in one of the variables, as highlighted for the previously mentioned acetyltransferase (ID 191), in which the percentage identity was $29.3 \%$, and the coverage $81 \%$.

Another bias may stem from the sequencing technology (454/Roche Genome Sequencer) and the gene prediction method used for both genomes; other approaches may have been used in the other six genomes screened. A particular example is the case of two orthologues (ID 48 and 49) that correspond to a single gene that was artificially split in two different coding sequences. After manual visualization of the sequencing data from both genomes, it appeared that frameshifts were caused by two homopolymer errors due to the sequencing technology at similar positions in the two bacteria. This artificially split gene encodes an ATPdependent Clp protease $(\mathrm{ClpB})$ that is, in fact, present in three species: L. pneumophila, C. trachomatis and Pr. amoebophila, although it was considered to be absent from the six genomes screened.

In addition, a number of proteins that are considered orthologues in L. drancourtii and P. acanthamoebae do not share a common history. In these cases, BLAST searches retrieved proteins from completely different organisms and the phylogenies clearly showed a divergent history, with the Parachlamydia and Legionella hits being grouped in distinct clusters (e.g., ID 301, Additional file 10, Figure S2). The phylogenies exhibiting this topology are easily identifiable and, therefore, do not cause a bias in the results. One option to avoid misidentifying orthologues would have been to perform a BLAST comparison versus the non-redundant database and to assign orthology only if the gene occurred within the best BLAST hits.

We did not identify any gene transfers from Chlamydiales to L. drancourtii. However, this finding may be due to the limited number of genomic sequences from Chlamydia-related bacteria available in the non-redundant database. Indeed, most published genomes belong to the family Chlamydiaceae, which includes bacteria that are highly adapted to their non-amoebal eukaryotic hosts and exhibit small genomes that typically range from $1 \mathrm{Mbp}$ to $1.2 \mathrm{Mbp}$. Additionally, the Chlamydiales order presents a biphasic developmental cycle the relatively inactive extracellular form is surrounded by a highly cross-linked extracellular matrix [35] that might relatively non-permissive to genetic exchanges. The replicating form, possessing a larger size and reduced extracellular matrix, might be more permeable to such exchanges that should subsequently arise within the host inclusion. The ability of Chlamydia-related bacteria to grow within amoebae would thus facilitate gene exchanges with other amoeba-infecting microorganisms. This hypothesis would be strengthened by in vitro evidence of co-localisation in amoebae of $P$. acanthamoebae, or any other amoeba-resistant chlamydiae, with Legionella.

Ogata et al. [19] suggested that gene exchanges among amoeba-resistant bacteria are an important mechanism for triggering the evolution of intracellular microorganisms. The recent analysis of the Amoebophilus asiaticus genome identified a large prevalence of gene transfer events among amoeba-resistant bacteria [10]. In our study, several phylogenies indicated possible HGTs between amoeba-associated bacteria (Legionella and Parachlamydia) and arthropod-associated bacteria, such as Rickettsia, Orientia, Wolbachia, and Ehrlichia. The presence of these genes in insect and arthropod endosymbionts suggests the possibility of intra-insect transfer because the Chlamydiales order also comprises the genera Fritschea and Rhabdochlamydia, which feature members that can infect arthropods [36-39]. Thus, as proposed by Thomas et al. [40], gene exchanges might be a common mechanism in intracellular bacteria that exhibit a sympatric lifestyle and may occur not only between amoebal symbionts but also between those found in arthropods. In addition, we identified a significant BLAST match with Mimivirus, a large amoebainfecting virus, for a hypothetical protein (ID 404), thereby supporting the hypothesis that intra-amoebal viruses are also capable of genetic exchanges with other microorganisms found in this environment [7].

In most cases, the Legionella or Parachlamydia proteins were largely diverged from their closest neighbors, suggesting that the gene transfers were evolutionarily ancient events. The inclusion of the best BLAST hits vs NR in the phylogenetic reconstruction identified the putative partner of transfer among all available sequenced genomes. As an example, the phylogenetic reconstruction of the PEBP family protein (ID:208) clustered $L$. drancourtii with the amoebal symbiont Amoebophilus asiaticus (Table 2). Due to the lack of sequenced genomes in species closely-related to $L$. drancourtii or P. acanthamoebae, the direction of the transfer and the period of exchange relative to bacterial 
speciation cannot be unequivocally established. There is no strong evidence for recent HGT events that can enable us to pinpoint the exact partners of the transfer, and an intermediate host that has not been sequenced yet might be implicated in the future.

No genes were surrounded on both sides by IS but, since no recent HGT was identified, insertion sequences might have diverged over time sufficiently to impair their identification. The close GC content values between the L. drancourtii and P. acanthamoebae species and the large divergence in the GC content among genes prevent us from confirming gene exchanges between these two partners by this method. Moreover, the large range of GC content within a bacterial order makes the use of this measure difficult for discriminating a potential origin of transfer because the phylogenetic tree usually shows distant relationships between our candidate partners.

\section{Conclusion}

This study presents a first genome-scale analysis that provides evidence for numerous HGT that occurred among three major intra-amoebal bacterial phyla. Moreover, this article reports a new genome of an amoebal pathogen, $L$. drancourtii, that opens new possibilities for the investigation of such important horizontal transfer events and provides data for researchers interested in the biology of this species. With the availability of additional genome sequences, a complete study of all sequenced intra-amoebal genomes would be of large interest and will bring in the future a more complete picture of gene exchanges occurring between amoebaresistant microorganisms. As previously suggested [7], the amoebae probably have a major role in bringing amoeba-associated microorganisms close enough to facilitate genetic exchanges. The occurrence of HGT between intra-amoebal pathogens is of importance, since such gene exchanges may play an important role in the selection of bacteria able to resist both the amoebae and other phagocytic cells such as the macrophages, which thus represent good candidates as new pathogens

\section{Methods}

\section{Study design}

To study the occurrence of gene transfer between amoebal pathogens, we sequenced the genome of the L. drancourtii strain LLAP-12 and compared it to the draft genome of the $P$. acanthamoebae strain Hall's coccus. Details on the dirty genome sequencing method used for $L$. drancourtii are provided with the Supplementary Materials (Additional file 1). After identifying orthologous genes present in both the $L$. drancourtii and $P$. acanthamoebae genomes, we searched for homologous genes in six bacterial genomes and one amoebal genome, as detailed below. The presence or absence of every orthologue in each clade was used to infer a heat map and a score. The corresponding clusters were investigated in more details. In addition, selected genes were compared by BLAST [41] to the non-redundant (nr) GenBank database and subjected to phylogenetic analyses.

\section{Identification of conserved genes}

To identify genes that were present in both L. drancourtii and P. acanthamoebae, we performed BLASTP comparisons between both genomes and the best reciprocal hits were retrieved if they exhibited a similarity $>30 \%$ and a query and hit length coverage $>60 \%$. These conserved genes were searched for in one amoebal genome (Dictyostelium discoideum AX4 [NC_007087$\mathrm{NC}$ _007092], the only fully sequenced amoebal genome available to date) and six bacterial genomes with a decreasing degree of phylogenetic relatedness respectively to $L$. drancourtii and P. acanthamoebae. Model organisms or medically important bacteria were selected within the same family (Legionella pneumophila Paris [NC_006368], respectively Pr. amoebophila UWE25 [NC_005861]), within the same order (Coxiella burnetii RSA 493 [NC_002971], respectively Chlamydia trachomatis D/UW-3/CX [NC_000117]) and within the same phylum (Escherichia coli K12 substr. MG1655 [NC_000913.2], respectively Rhodopirellula baltica SH1 [NC_005027]). Each gene was considered present if it exhibited $>30 \%$ identity and had a length coverage $>$ $60 \%$ on both the query and hit. The genes were classified according to the number of hits obtained using $P$. acanthamoebae or L. drancourtii as the query against each of the six bacterial genomes based on a score of 0 (no hits in any $\mathrm{f}$ the screened genomes) to 6 (hits in all of the screened bacterial genomes).

\section{Gene clustering and the definition of core genes}

Hierarchical clustering was performed based on the Euclidean distance of the presence or absence of each gene in the studied genomes. Using the $\mathrm{H}$-clust function (an in-house R script), super clusters were defined at a dendrogram height equal to 3 . The clusters were represented on a heat map drawn using an in-house $\mathrm{R}$ script [42]. Core genes were defined as any gene present in at least 4 of the 6 screened genomes (score $\geq 4$ ) and were not further analyzed.

\section{BLAST and phylogenetic analyses}

A BLASTP search against the nr database http://blast. ncbi.nlm.nih.gov/Blast.cgi was performed for genes with scores between 0 and 3 . For each gene, a phylogenetic analysis was conducted with the 20 best BLASTP hits of $P$. acanthamoebae and $L$. drancourtii that was restricted 
to one representative species per genus. Sequence alignment was performed using Muscle [43]. Phylogenetic relationships among species were inferred with PhyML (PHYlogenetic inferences using Maximum Likelihood) [44] using the WAG model. For particularly interesting cases, a phylogeny based on a Bayesian inference was performed using MrBayes [45] on alignments curated by Gblocks [46].

\section{Identification of lateral gene transfer}

Genes exhibiting a topology different from that expected for core genes were classified according to one of the five following topologies: i) $P$. acanthamoebae genes clustering with $L$. drancourtii; ii) P. acanthamoebae genes clustering with Legionellales; iii) Legionellales genes clustering with Chlamydiales; iv) P. acanthamoebae and/or L. drancourtii genes clustering with Rickettsia, Ehrlichia and Wolbachia and (v) genes matching eukaryotic proteins by BLAST homology searches.

To characterize further the genes exhibiting an unexpected phylogenetic topology, their GC content was examined relative to the genomic mean with $R$ [42] using the seqinR package [47]. A BLASTN comparison was achieved versus ISfinder [48] with an e-value of 1e5 to search for insertion sequences in the genomes of $L$. drancourtii and P. acanthamoebae.

\section{Additional material}

Additional file 1: Supplemental data.

Additional file 2: Table S1. Conserved orthologous genes. This table lists the 208 orthologues of L. drancourtii (LLAP) and Pr. acanthamoebae $(\mathrm{PAH})$ also found in the genomes of Pr. amoebophila, C. trachomatis, $R$. baltica, L. pneumophila, C. burnetii, and E. coli.

Additional file 3: Table S2. Orthologous genes identified in $\mathbf{5}$ bacteria. This table presents the 80 orthologues of L. drancourtii (llap) and $P$. acanthamoebae (pah) identified in five among the six bacterial genomes screened (Pr. amoebophila, C. trachomatis, R. baltica, L. pneumophila, C. burnetii, and E. coli). The last column indicates the bacterial genome where the corresponding gene could not be identified.

Additional file 4: Table S3. Orthologous genes identified in four bacteria. Accession number and annotation of the 77 orthologues of $L$. drancourtii (LLAP) and P. acanthamoebae (PAH) also identified in four of the six bacterial genomes screened (Pr. amoebophila, C. trachomatis, R. baltica, L. pneumophila, C. burnetii, and E. coli)

Additional file 5: Table S4. Orthologous genes identified in three bacteria. Accession number and annotation of the 59 orthologues of $L$. drancourtii (LLAP) and P. acanthamoebae (PAH) also identified in three of the six bacterial genomes screened (Pr. amoebophila, C. trachomatis, $R$. baltica, L. pneumophila, C. burnetii, and E. coli).

Additional file 6: Table S5. Orthologous genes identified in two bacteria. Accession number and annotation of the 37 orthologues of $L$. drancourtii (LLAP) and P. acanthamoebae (PAH) also detected in two among the six bacterial genomes screened (Pr. amoebophila, C. trachomatis, R. baltica, L. pneumophila, C. burnetii, and E. coli). The last column indicates the two bacterial species that harbour the corresponding homologous gene.

Additional file 7: Table S6. Orthologous genes identified in one single bacterium. Accession number and annotation of the 27 orthologues of L. drancourtii (llap) and P. acanthamoebae (pah) identified in only one of the six bacterial genomes screened (Pr. amoebophila, C. trachomatis, R. baltica, L. pneumophila, C. burnetii, and E. coli). The last column indicates the bacterial species that harbours the corresponding homologous gene.

Additional file 8: Table S7. Orthologous genes not identified in any bacteria screened. Accession number and annotation of the 20 orthologues of L. drancourtii (LLAP) and P. acanthamoebae (PAH) that could not be detected in any of the six bacterial genomes screened $\operatorname{Pr}$. amoebophila, C. trachomatis, R. baltica, L. pneumophila, C. burnetii, and E. coli).

Additional file 9: Figure S1. Phylogenetic trees clustering $L$. drancourtii and $P$. acanthamoebae. Maximum likelihood trees of $L$. drancourtii and $P$. acanthamoebae orthologous proteins and their 20 best blast hits, restricted to one representative per genus. In these phylogenetic reconstruction, $L$. drancourtii and $P$. acanthamoebae cluster together. Sequences retrieved by using $L$. drancourtii or $P$. acanthamoebae protein as a query, are indicated with the prefix LLAP or $\mathrm{PAH}$, respectively. Bacteria belonging to the Legionellales, Chlamydiales and Rickettsiales are shown respectively in blue, green and red.

Additional file 10: Figure S2. Phylogenetic trees clustering $P$. acanthamoebae and Legionellales. Maximum likelihood trees of $L$. drancourtii and $P$. acanthamoebae orthologous proteins and their 20 best blast hits, restricted to one representative per genus. (A-O) $P$. acanthamoebae directly branches with Legionellales and (O-R) P. acanthamoebae clusters with L. pneumophila but more distantly to other Legionellales. Sequences retrieved by using $L$. drancourtii or $P$. acanthamoebae protein as a query, are indicated with the prefix LLAP or $\mathrm{PAH}$, respectively. Bacteria belonging to the Legionellales, Chlamydiales and Rickettsiales are shown respectively in blue, green and red.

Additional file 11: Figure S3. Phylogenetic trees clustering Chlamydiales and Legionellales. Maximum likelihood trees of $L$. drancourtii and $P$. acanthamoebae orthologous proteins and their 20 best blast hits, restricted to one representative per genus, where bacteria of the Chlamydiales order and the Legionellales are directy related. Sequences retrieved by using $L$. drancourtii or P.acanthamoebae protein as a query, are indicated with the prefix LLAP or PAH, respectively. Bacteria belonging to the Legionellales, Chlamydiales and Rickettsiales orders are shown respectively in blue, green and red.

Additional file 12: Figure S4. Phylogenetic trees clustering $L$. drancourtii or P. acanthamoebae with Rickettsiales and A. asiaticus. Maximum likelihood trees of $L$. drancourtii and $P$. acanthamoebae orthologous proteins and their 20 best blast hits, restricted to one representative per genus, where Chlamydiales or Legionellales representatives are directy related to other intracellular bacteria such as Rickettsia, Ehrlichia, Orientia or Wolbachia. In the last phylogenetic reconstruction Legionella clusters with another intra-amoebal bacterium, Amoebophilus asiaticus. Trees for ID 191, 192 and 387 are found in Additional file 2, Figure S1 and the tree for ID 263 is shown in Additional file 4, Figure S3. Sequences retrieved by using $L$. drancourtii or $P$. acanthamoebae protein as a query, are indicated with the prefix LLAP or $\mathrm{PAH}$, respectively. Bacteria belonging to the Legionellales, Chlamydiales and Rickettsiales orders are shown respectively in blue, green and red.

Additional file 13: Figure S5. Phylogenetic trees with eukaryotic representatives. In two cases (A and C), eukaryotic sequences were identified by BLASTP homology searches. In another tree (B), L. drancourtii clustered with the amoeba-associated Bacteroidetes named Amoebophilus asiaticus. Maximum likelihood trees of $L$. drancourtii and $P$. acanthamoebae orthologous proteins were build using their 20 best blast hits, restricted to one representative per genus. Sequences retrieved by using $L$. drancourtii or $P$. acanthamoebae protein as a query, are indicated with the prefix LLAP or PAH, respectively. Bacteria belonging to the Legionellales, Chlamydiales and Rickettsiales orders are shown respectively in blue, green and red.

Additional file 14: Figure S6. Genic GC content. The genic GC contents of $P$. acanthamoebae (A) and L. drancourtii (B) are shown in grey, whereas orthologous genes are shown in light pink. Horizontally transferred genes are colored by categories of the putative partners 
according to the legend within the figure: in blue-green between $L$. drancourtii and P. acanthamoebae; in purple between Legionellales and Chlamydiales members; in pink between $L$. drancourtii or $P$. acanthamoebae and Rickettsiales; in yellow with Eukaryotes or A. asiaticus. Panels (C) and (D) present the genic GC content at the 3rd position of the codon respectively in $P$. acanthamoebae and $L$. drancourtii using a similar color-code.

Additional file 15: Figure S7. Percentage identity and coverage in $L$. drancourtii and P. acanthamoebae orthologues. Proteins potentially transferred horizontally are colored according to four categories of gene transfer: in blue-green between L. drancourtii and P. acanthamoebae; in purple between Legionellales and Chlamydiales members; in pink between L. drancourtii or P. acanthamoebae and Rickettsiales; in yellow with Eukaryotes or A. asiaticus.

\section{List of Abbreviations}

IS: insertion sequence; HGT: horizontal gene transfer; LLAP: Legionella-like amoebal pathogen 12; NR: non-redundant; ORF: Open Reading Frame; PAH: Parachlamydia acanthamoebae strain Hall's coccus; PhyML: PHYlogenetic inferences using Maximum Likelihood; T4SS: Type IV secretion system.

\section{Acknowledgements}

G. Greub is supported by the Leenards Foundation through a career award titled "Bourse Leenards pour la relève académique en médecine clinique à Lausanne".

\section{Author details}

'Unité des rickettsies, Faculté de Médecine, Université de la Méditerranée, Marseille, France URMITE CNRS-IRD UMR 6236, Faculté de Médecine, 27 boulevard Jean Moulin, 13385 Marseille Cedex 05, France. ${ }^{2}$ Center for Research on Intracellular Bacteria, Institute of Microbiology, University of Lausanne and University Hospital, Bugnon 48, 1011 Lausanne, Switzerland.

\section{Authors' contributions}

PEF and GGr conceived and designed the study. DR participated in the study design. GGi and CB performed the sequence comparison and phylogenetic analyses. CM made the LLAP genome draft annotation. CR performed the genome sequencing. GGi, CB, PEF and GGr analyzed the data and wrote the manuscript. All authors revised the final version of the manuscript.

\section{Competing interests}

The authors declare that they have no competing interests.

Received: 5 May 2011 Accepted: 2 November 2011

Published: 2 November 2011

\section{References}

1. Clarholm M: Bacteria and protozoa as integral components of the forest ecosystem-their role in creating a naturally varied soil fertility. Antonie Van Leeuwenhoek 2002, 81(1-4):309-318.

2. Aderem A, Underhill DM: Mechanisms of phagocytosis in macrophages. Annu Rev Immunol 1999, 17:593-623.

3. Cardelli J: Phagocytosis and macropinocytosis in Dictyostelium: phosphoinositide-based processes, biochemically distinct. Traffic 2001, 2(5):311-320.

4. Greub G, La Scola B, Raoult D: Amoebae-resisting bacteria isolated from human nasal swabs by amoebal coculture. Emerg Infect Dis 2004, 10(3):470-477.

5. Birtles RJ, Rowbotham TJ, Raoult D, Harrison TG: Phylogenetic diversity of intra-amoebal legionellae as revealed by 165 rRNA gene sequence comparison. Microbiology 1996, 142(Pt 12):3525-3530.

6. Greub G, Raoult D: Parachlamlydiaceae: Potential emerging pathogens. Emerging Infectious Diseases 2002, 8(6):625-630.

7. Moliner C, Fournier PE, Raoult D: Genome analysis of microorganisms living in amoebae reveals a melting pot of evolution. FEMS Microbiol Rev 2010.
8. Greub G, Raoult D: Microorganisms resistant to free-living amoebae. Clin Microbiol Rev 2004, 17(2):413-433.

9. Casadevall A: Evolution of intracellular pathogens. Annu Rev Microbiol 2008, 62:19-33.

10. Schmitz-Esser S, Tischler P, Arnold R, Montanaro J, Wagner M, Rattei T, Horn M: The genome of the amoeba symbiont "Candidatus Amoebophilus asiaticus" reveals common mechanisms for host cell interaction among amoeba-associated bacteria. J Bacteriol 2010, 192(4):1045-1057.

11. Horn M, Collingro A, Schmitz-Esser S, Beier CL, Purkhold U, Fartmann B, Brandt $P$, Nyakatura GJ, Droege M, Frishman D, et al: llluminating the evolutionary history of chlamydiae. Science 2004, 304(5671):728-730

12. Greub G, Kebbi-Beghdadi C, Bertelli C, Collyn F, Riederer BM, Yersin C, Croxatto A, Raoult D: High throughput sequencing and proteomics to identify immunogenic proteins of a new pathogen: the dirty genome approach. PLoS One 2009, 4(12):e8423.

13. Bertelli C, Collyn F, Croxatto A, Ruckert C, Polkinghorne A, KebbiBeghdadi C, Goesmann A, Vaughan L, Greub G: The Waddlia genome: a window into chlamydial biology. PLoS One 2010, 5(5):e10890.

14. Cazalet C, Rusniok C, Bruggemann H, Zidane N, Magnier A, Ma L, Tichit M, Jarraud S, Bouchier C, Vandenesch F, et al: Evidence in the Legionella pneumophila genome for exploitation of host cell functions and high genome plasticity. Nat Genet 2004, 36(11):1165-1173.

15. Chien M, Morozova I, Shi S, Sheng H, Chen J, Gomez SM, Asamani G, Hill K, Nuara J, Feder $\mathrm{M}$, et al: The genomic sequence of the accidental pathogen Legionella pneumophila. Science 2004, 305(5692):1966-1968.

16. D'Auria G, Jimenez-Hernandez N, Peris-Bondia F, Moya A, Latorre A: Legionella pneumophila pangenome reveals strain-specific virulence factors. BMC Genomics 2010, 11(1):181.

17. Glockner G, Albert-Weissenberger C, Weinmann E, Jacobi S, Schunder E, Steinert M, Hacker J, Heuner K: Identification and characterization of a new conjugation/type IVA secretion system (trb/tra) of Legionella pneumophila Corby localized on two mobile genomic islands. Int I Med Microbiol 2008, 298(5-6):411-428.

18. Kozak NA, Buss M, Lucas CE, Frace M, Govil D, Travis T, Olsen-Rasmussen M, Benson RF, Fields BS: Virulence factors encoded by Legionella longbeachae identified on the basis of the genome sequence analysis of clinical isolate D-4968. J Bacteriol 2010, 192(4):1030-1044.

19. Ogata H, La Scola B, Audic S, Renesto P, Blanc G, Robert C, Fournier PE, Claverie JM, Raoult D: Genome sequence of Rickettsia bellii illuminates the role of amoebae in gene exchanges between intracellular pathogens. Plos Genetics 2006, 2(5):733-744.

20. La Scola B, Birtles RJ, Greub G, Harrison TJ, Ratcliff RM, Raoult D: Legionella drancourtii sp. nov., a strictly intracellular amoebal pathogen. Int J Syst Evol Microbiol 2004, 54(Pt 3):699-703.

21. Wirz M, Polkinghorne A, Dumrese C, Ziegler U, Greub G, Pospischil A, Vaughan L: Predator or prey? Chlamydophila abortus infections of a freeliving amoebae, Acanthamoeba castellani 9GU. Microbes Infect 2008, 10(6):591-597.

22. Andersson SG, Kurland CG: Reductive evolution of resident genomes. Trends Microbiol 1998, 6(7):263-268.

23. Moran NA, Plague GR: Genomic changes following host restriction in bacteria. Curr Opin Genet Dev 2004, 14(6):627-633.

24. Greub G: Parachlamydia acanthamoebae, an emerging agent of pneumonia. Clinical Microbiology and Infection 2009, 15(1):18-28.

25. Alvarez-Martinez CE, Christie PJ: Biological diversity of prokaryotic type IV secretion systems. Microbiol Mol Biol Rev 2009, 73(4):775-808.

26. Juhas M, Crook DW, Hood DW: Type IV secretion systems: tools of bacterial horizontal gene transfer and virulence. Cell Microbiol 2008, 10(12):2377-2386.

27. Lawley TD, Klimke WA, Gubbins MJ, Frost LS: F factor conjugation is a true type IV secretion system. FEMS Microbiol Lett 2003, 224(1):1-15.

28. Greub G, Collyn F, Guy L, Roten CA: A genomic island present along the bacterial chromosome of the Parachlamydiaceae UWE25, an obligate amoebal endosymbiont, encodes a potentially functional F-like conjugative DNA transfer system. Bmc Microbiology 2004, 4.

29. Segal G, Feldman M, Zusman T: The Icm/Dot type-IV secretion systems of Legionella pneumophila and Coxiella burnetii. FEMS Microbiol Rev 2005, 29(1):65-81.

30. Gil R, Silva FJ, Pereto J, Moya A: Determination of the core of a minimal bacterial gene set. Microbiol Mol Biol Rev 2004, 68(3):518-537. 
31. Gentschev I, Dietrich G, Goebel W: The E. coli alpha-hemolysin secretion system and its use in vaccine development. Trends Microbiol 2002, 10(1):39-45.

32. Moliner C, Raoult D, Fournier PE: Evidence that the intra-amoebal Legionella drancourtii acquired a sterol reductase gene from eukaryotes. BMC Res Notes 2009, 2:51.

33. Moliner C, Raoult D, Fournier PE: Evidence of horizontal gene transfer between amoeba and bacteria. Clin Microbiol Infect 2009, 15(Suppl 2):178-180.

34. Lawrence JG, Ochman $\mathrm{H}$ : Amelioration of bacterial genomes: rates of change and exchange. J Mol Evol 1997, 44(4):383-397.

35. McCoy AJ, Maurelli AT: Building the invisible wall: updating the chlamydial peptidoglycan anomaly. Trends Microbiol 2006, 14(2):70-77.

36. Everett KDE, Thao ML, Horn M, Dyszynski GE, Baumann P: Novel chlamydiae in whiteflies and scale insects: endosymbionts 'Candidatus Fritschea bemisiae' strain Falk and 'Candidatus Fritschea eriococci' strain Elm. International Journal of Systematic and Evolutionary Microbiology 2005, 55:1581-1587.

37. Thao ML, Baumann L, Hess JM, Falk BW, Ng JC, Gullan PJ, Baumann P: Phylogenetic evidence for two new insect-associated Chlamydia of the family Simkaniaceae. Curr Microbiol 2003, 47(1):46-50.

38. Corsaro D, Thomas V, Goy G, Venditti D, Radek R, Greub G: 'Candidatus Rhabdochlamydia crassificans', an intracellular bacterial pathogen of the cockroach Blatta orientalis (Insecta: Blattodea). Systematic and Applied Microbiology 2007, 30(3):221-228.

39. Kostanjsek R, Strus J, Drobne D, Avgustin G: 'Candidatus Rhabdochlamydia porcellionis', an intracellular bacterium from the hepatopancreas of the terrestrial isopod Porcellio scaber (Crustacea: Isopoda). Int J Syst Evol Microbiol 2004, 54(Pt 2):543-549.

40. Thomas V, Greub G: Amoeba/amoebal symbiont genetic transfers: lessons from giant virus neighbours. Intervirology 2010, 53(5):254-267.

41. Altschul SF, Madden TL, Schaffer AA, Zhang J, Zhang Z, Miller W, Lipman DJ: Gapped BLAST and PSI-BLAST: a new generation of protein database search programs. Nucleic Acids Res 1997, 25(17):3389-3402.

42. Team RDC: R: A Language and Environment for Statistical Computing. 2010.

43. Edgar RC: MUSCLE: a multiple sequence alignment method with reduced time and space complexity. Bmc Bioinformatics 2004, 5:113.

44. Guindon S, Gascuel O: A simple, fast, and accurate algorithm to estimate large phylogenies by maximum likelihood. Syst Biol 2003, 52(5):696-704.

45. Ronquist F, Huelsenbeck JP: MrBayes 3: Bayesian phylogenetic inference under mixed models. Bioinformatics 2003, 19(12):1572-1574.

46. Talavera G, Castresana J: Improvement of phylogenies after removing divergent and ambiguously aligned blocks from protein sequence alignments. Syst Biol 2007, 56(4):564-577.

47. Charif D, Lobry JR: SeqinR 1.0-2: a contributed package to the R project for statistical computing devoted to biological sequences retrieval and analysis. In Structural approaches to sequence evolution: Molecules, networks, populations. Edited by: Bastolla U, Porto M, Roman HE, Vendruscolo M. New York: Springer Verlag; 2007:207-232.

48. Siguier P, Perochon J, Lestrade L, Mahillon J, Chandler M: ISfinder: the reference centre for bacterial insertion sequences. Nucleic Acids Res 2006, , 34 Database: D32-36.

\section{doi:10.1186/1471-2164-12-542}

Cite this article as: Gimenez et al:: Insight into cross-talk between intraamoebal pathogens. BMC Genomics 2011 12:542.

\section{Submit your next manuscript to BioMed Central and take full advantage of:}

- Convenient online submission

- Thorough peer review

- No space constraints or color figure charges

- Immediate publication on acceptance

- Inclusion in PubMed, CAS, Scopus and Google Scholar

- Research which is freely available for redistribution

Submit your manuscript at www.biomedcentral.com/submit
Biomed Central 